\title{
Verzeichnis der Abkürzungen und der abgekürzt zitierten Literatur
}

a.A.

a.a.O.

ABBV

$\mathrm{ABE}$

$\mathrm{ABG}$

ABGB

abgedr.

ABGF

Abk.

abl.

ABl.

ABMG

$\mathrm{ABN}$

ABRK

ABRV

ABS

Abs.

Abschlussbericht

Abschn.

$\mathrm{ABU}$

ABVerm

abw.

AcP

a.E.

AEB

ÄndG

ÄndVO

AERB

AEUV

AFB

a.F.

AG

AGG

AGBG

AGlB

AHagB

$\mathrm{AHB}$

$\mathrm{AKB}$

AktG

anderer Ansicht

am angegebenen Ort

Allgemeine Bedingungen für die Baubestandsversicherung

Allgemeine Bedingungen für die Elektronikversicherung

Allgemeine Bedingungen für die Kaskoversicherung von Baugeräten

Allgemeines Bürgerliches Gesetzbuch (Österreich)

abgedruckt

Allgemeine Bedingungen für die dynamische Sachversicherung des Gewerbes und der Freien Berufe

Abkommen

ablehnend

Amtsblatt

Allgemeine Bedingungen für die Maschinen- und Kasko-Versicherung von fahrbaren und transportablen Geräten

Allgemeine Bedingungen für die Bauleistungsversicherung von Gebäudeneubauten durch Auftraggeber

Allgemeine Bedingungen für die Reparaturkosten von Kraftwagen

Allgemeine Bedingungen für die Reiserücktrittskostenversicherung

Allgemeine Bedingungen für die Sachversicherung (Österreich)

Absatz

siehe KomE

Abschnitt

Allgemeine Bedingungen für die Bauleistungsversicherung von Unternehmerleistungen

Allgemeine Bedingungen für die Vermögenshaftpflichtversicherung

abweichend

Archiv für civilistische Praxis (zit. nach Band, Jahr u. Seite)

am Ende

Allgemeine Einbruchdiebstahlversicherungsbedingungen

Änderungsgesetz

Änderungsverordnung

Allgemeine Bedingungen für die Einbruchdiebstahl- und Raubversicherung

Vertrag über die Arbeitsweise der Europäischen Union

Allgemeine Bedingungen für die Feuerversicherung

alte Fassung

Amtsgericht; Aktiengesellschaft

Allgemeines Gleichbehandlungsgesetz

Gesetz zur Regelung des Rechts der Allgemeinen Geschäftsbedingungen (AGB-Gesetz)

Allgemeine Bedingungen für die Glasversicherung

Allgemeine Hagelversicherungsbedingungen

Allgemeine Versicherungsbedingungen für die Haftpflichtversicherung Allgemeine Bedingungen für die Kfz-Versicherung

Gesetz über Aktiengesellschaften und Kommanditgesellschaften auf Aktien 
ALB

allg.

allg.M.

Alt.

AltZertG

a.M.

$\mathrm{AMB}$

AMBUB

AMG

$\mathrm{AMoB}$

amtl. Begr.

Anh.

Anl.

Anm.

AnwBl.

AnwKom

ao

AO

ARB

Art.

AStB

AT

AtomG

AUB

Auff.

Aufl.

AuR

ausdrückl.

ausführl.

AusfVO

ausl.

AuslG

AuslKfzPflVV

AuslPflVG

Auslunf

AusnVO

ausschl.

Ausschussbericht

AV

AVB

AVB-AVG

AVB MaV

AVBR

AVBSP
Allgemeine Bedingungen für die kapitalbildende Lebensversicherung allgemein

allgemeine Meinung

Alternative

Gesetz über die Zertifizierung von Altersvorsorgeverträgen

anderer Meinung

Allgemeine Maschinen-Versicherungsbedingungen; ab 2008: Allgemeine Bedingungen für die Maschinenversicherung von stationären Maschinen

Allgemeine Maschinen-Betriebsunterbrechungs-Versicherungsbedingungen

Arzneimittelgesetz

Allgemeine Montageversicherungsbedingungen

amtliche Begründung

Anhang

Anlage

Anmerkung

Anwaltsblatt

siehe NK-BGB

außerordentlich

Abgabenordnung

Allgemeine Bedingungen für die Rechtsschutzversicherung

Artikel

Allgemeine Bedingungen für die Sturmversicherung

Allgemeiner Teil

Gesetz über die friedliche Verwendung der Kernenergie und den Schutz gegen ihre Gefahren (Atomgesetz)

Allgemeine Unfallversicherungsbedingungen

Auffassung

Auflage

Arbeit und Recht

ausdrücklich

ausführlich

Ausführungsverordnung

ausländisch

Ausländergesetz

Verordnung über die Kraftfahrzeug-Haftpflichtversicherung ausländischer Kraftfahrzeuge und Kraftfahrzeuganhänger

Gesetz über die Haftpflichtversicherung für ausländische Kraftfahrzeuge und Kraftfahrzeuganhänger

Unfälle mit Auslandsbezug

Ausnahmeverordnung

ausschließlich

Beschlussempfehlung und Bericht des Rechtsausschusses zum Regierungsentwurf eines Gesetzes zur Reform des Versicherungsvertragsrechts (BTDrucks. 16/5862)

Allgemeine Verfügung

Allgemeine Versicherungsbedingungen

Allgemeine Versicherungsbedingungen für die VermögensschadenHaftpflichtversicherung von Aufsichtsräten, Vorständen und Geschäftsführern

Allgemeine Bedingungen für die Versicherung von Maschinen, maschinellen Einrichtungen und Apparaten

Allgemeine Bedingungen für die Versicherung von Reisegepäck

Allgemeine Bedingungen für die Versicherung von Juwelen, Schmuckund Pelzsachen im Privatbesitz 
AVB Vermögen

AVBW

AVFE

AVFEBU

\section{AVFEM}

AVG

AVP

AVR

AVSZ

AVTHK

AWaB

AWB

AWG

Az.

\section{Bach/Moser}

BaFin

BAG

Bamberger/Roth/Bearbeiter

BAnz.

Basedow/Fock

Baner

BauGB

BAV (BAA)

BayGaStellv

BayObLG

$\mathrm{BB}$

BBG

BBR

BBR ITD

Bd.

BDSG

Bearb.

Beckmann/Matusche-

Beckmann/Bearbeiter

BeckOK-BGB

BeckRS

begl.

Begr.
Allgemeine Versicherungsbedingungen zur Haftpflichtversicherung für Vermögensschäden

Allgemeine Bedingungen für die Kasko-Versicherung von Wassersportfahrzeugen

Allgemeine Versicherungsbedingungen für Fernmelde- und sonstige elektronische Anlagen

Allgemeine Betriebsunterbrechungs-Bedingungen bei Fernmelde- und sonstigen elektrotechnischen Anlagen

Allgemeine Bedingungen für die Mehrkostenversicherung bei Fernmeldeanlagen und sonstigen elektrotechnischen Anlagen

Angestelltenversicherungsgesetz

Allgemeine Bedingungen für die Versicherung von Pferden und anderen Einhufern

Allgemeine Bedingungen für die Versicherung von Rindern

Allgemeine Bedingungen für die Versicherung von Schweinen, Schafen und Ziegen

Allgemeine Bedingungen für die Tierkrankenversicherung von Hunden und Katzen

Allgemeine Versicherungsbedingungen für die Waldbrandversicherung

Allgemeine Bedingungen für die Leitungswasserversicherung

Außenwirtschaftsgesetz

Aktenzeichen

Private Krankenversicherung, $\mathrm{MB} / \mathrm{KK}$ - und $\mathrm{MB} / \mathrm{KT}$-Kommentar, 5. Aufl. (2015)

Bundesanstalt für Finanzdienstleistungsaufsicht

Bundesarbeitsgericht bzw. Bundesamt für Güterverkehr

Kommentar zum Bürgerlichen Gesetzbuch in vier Bänden, 4. Aufl. (2018)

Bundesanzeiger

Europäisches Versicherungsvertragsrecht, Bd. I-III (2002/03)

Die Kraftfahrtversicherung, 6. Aufl. (2010)

Baugesetzbuch

Bundesaufsichtsamt für das Versicherungs- (bis 1973) und Bausparwesen (bis 2001)

Bayerische Garagen- und Stellplatzverordnung

Bayerisches Oberstes Landesgericht

Der Betriebs-Berater

Bundesbeamtengesetz

Besondere Bedingungen und Risikobeschreibungen

Besondere Bedingungen und Risikobeschreibungen für die Haftpflichtversicherung von IT-Dienstleistern

Band

Bundesdatenschutzgesetz

Bearbeitung

Versicherungsrechts-Handbuch, 3. Aufl. (2015)

Beck'scher Online-Kommentar BGB, hrsg. von Bamberger/Roth (Stand: 1.2.2017)

Elektronische Entscheidungsdatenbank in beck-online (zitiert mit Jahrgang und lfd. Nummer)

beglaubigt

Begründung

zum VVG: RTDrucks Nr. 364, 12. Legislaturperiode, 1. Session 1907; zum PflVersG v. 7.11.1939: DJ 39, 1771; 
Bek.

Bekl.

Bem.

Benkel/Hirschberg

ber.

Berliner Kommentar/

Bearbeiter

Berz/Burmann/Bearbeiter

bes.

BesBed Priv

Beschl.

Beschw.

Bespr.

Best.

bestr.

betr.

BeurkG

$\mathrm{BFH}$

BGB

BGBl.

$\mathrm{BGH}$

BGHGrS

BGHR

BGHSt

BGHZ

BHHJJ/Bearbeiter

BMI

BMJ

Böhme/Biela/Tomson

BR

BRAK

BRAO

BRDrucks.

BReg.

BRProt.

BRRG

Bruck Versicherungsvertrag

Bruck/Möller/Bearbeiter ${ }^{8}$ zur VO v. 19.12.1939: Amtl. Sonderveröffentl. d. DJ Nr. 20, Beilage zur DJ Nr. 3/1940;

zum G v. 28.12.1942: DJ 43, $41 \mathrm{ff}$;

zur VO v. 6.4.1943: DJ 43, 269;

zum G v. 5.4.1965 (PflVersG n.F.): BRDrucks. IV/2252 S $11 \mathrm{ff}$.

zum RegE VVGReformG v. 20.12.2006 BTDrucks. 16/3945

Bekanntmachung

Beklagter

Bemerkung

Lebens- und Berufsunfähigkeitsversicherung, ALB- und BUZ-Kommentar, 2. Aufl. (2011)

berichtigt

Berliner Kommentar zum Versicherungsvertragsgesetz: Kommentar zum deutschen und österreichischen VVG, hrsg. von H. Honsell (1999)

Handbuch des Straßenverkehrsrechts, hrsg. von Berz/Burmann, 38. Ergänzungslieferung (12/2017) besonders

Besondere Bedingungen und Risikobeschreibungen für die Privathaftpflichtversicherung

Beschluss

Beschwerde

Besprechung

Bestimmung

bestritten

betreffend

Beurkundungsgesetz

Bundesfinanzhof

Bürgerliches Gesetzbuch

Bundesgesetzblatt

Bundesgerichtshof

Bundesgerichtshof, Großer Senat

BGH-Rechtsprechung Zivilsachen

Entscheidungen des Bundesgerichtshofes in Strafsachen (zit. nach Band u. Seite)

Entscheidungen des Bundesgerichtshofes in Zivilsachen (zit. nach Band u. Seite)

Burmann/Heß/Hühnermann/Jahnke/Janker, Straßenverkehrsrecht, 25. Auflage (2018)

Bundesminister(ium) des Inneren

Bundesminister(ium) der Justiz

Kraftverkehrs-Haftpflicht-Schäden. Handbuch für die Praxis, 26. Aufl. (2018) (bis zur 22. Aufl. Becker/Böhme)

Bundesrat

Bundesrechtsanwaltskammer

Bundesrechtsanwaltsordnung

Bundesrats-Drucksache

Bundesregierung

Protokolle des Bundesrates

Beamtenrechtsrahmengesetz

Kommentar zum Reichsgesetz über den Versicherungsvertrag, 7. Aufl. (1932)

Kommentar zum Versicherungsvertragsgesetz und zu den Allgemeinen Versicherungsbedingungen unter Einschluss des Versicherungsvermittlerrechtes, 8. Aufl. (1961-2002) 
Bruck/Möller/Bearbeiter

Bruns,

Privatversicherungsrecht

BSG

BSHG

Bsp.

BStBl.

BT

BTDrucks.

BU

Buchst.

van Bühren/Bearbeiter $\mathrm{Hdb}$

van Bühren

van Bühren/Plate/Bearbeiter

Buschbell/Hering/Bearbeiter

BVerfG

BVerfGE

BVerfGG

BVerwG

BVerwGE

bzgl.

bzw.

ca.

CR

dagg.

DAR

DAV

DB

DBKG

ders.

dgl.

d.h.

dies.

Diff., diff.

Dig.

DIN

Diss.

DJ

DJT

DöV

D\&O

DR

DRechtsw.

DRiB

DRiG

DRiZ

Drucks.
Großkommentar zum Versicherungsvertragsgesetz und zu den Allgemeinen Versicherungsbedingungen, herausgegeben von Horst Baumann/Roland Michael Beckmann/Katharina Johannsen/Ralf Johannsen/Robert Koch, 9. Aufl. (2008ff.)

Privatversicherungsrecht (2015)

Bundessozialgericht

Bundessozialhilfegesetz

Beispiel

Bundessteuerblatt

Besonderer Teil, Bundestag

Bundestagsdrucksache

Betriebsunterbrechung

Buchstabe

Handbuch Versicherungsrecht, 7. Aufl. (2017)

Das versicherungsrechtliche Mandat, 5. Aufl. (2015)

Allg. Bedingungen für die Rechtsschutzversicherung: ARB, 3. Auflage (2013)

Handbuch Rechtsschutzversicherung, 6. Aufl. (2015)

Bundesverfassungsgericht

Entscheidungen des Bundesverfassungsgerichts (zit. nach Band u. Seite)

Gesetz über das Bundesverfassungsgericht

Bundesverwaltungsgericht

Entscheidungen des Bundesverwaltungsgerichts (zit. nach Band u. Seite)

bezüglich

beziehungsweise

circa

Computer und Recht

dagegen

Deutsches Autorecht

Deutscher Anwaltsverein; Deutsche Aktuarvereinigung

Der Betrieb

Deutsches Büro Grüne Karte e.V.

derselbe

dergleichen

das heißt

dieselbe(n)

Differenzierung, differenzierend

Digesta

Deutsche Industrie Norm

Dissertation

Deutsche Justiz

Deutscher Juristentag

Deutsche öffentlich-rechtliche Versicherung

Directors and Officers (Liability Insurance)

Deutsches Recht, Wochenausgabe (vereinigt mit Juristische Wochenschrift) (1931-1945)

Deutsche Rechtswissenschaft (1936-1943)

Deutscher Richterbund

Deutsches Richtergesetz

Deutsche Richterzeitung

Drucksache 
Verzeichnis der Abkürzungen und der abgekürzt zitierten Literatur

DS

DSB

DStrR

dt.

DTV-VHV

DVBl.

DVO

DZWIR

E

ebd.

ebso.

ECB

ECBUB

$\mathrm{ED}$

ed(s)

EG

EGBGB

EGGVG

EGV

EGVVG

ehem.

Einf.

eingeh.

einschl.

einschr.

Einl.

entgg.

Entsch.

entspr.

Entw.

ErfK/Bearbeiter

Erg.

ErgBd.

Erl.

Erman/Bearbeiter

Erw.

EStG

etc.

$\mathrm{EU}$

EuGH

EuGHE

EuGVVO

EuR

europ.

EUV

EuZW
Der Sachverständige

Datenschutzberater

Deutsches Steuerrecht

deutsch

DTV-Verkehrshaftungsversicherung

Deutsches Verwaltungsblatt

Durchführungsverordnung

Deutsche Zeitschrift für Wirtschaftsrecht

Entwurf bzw. Entscheidung

ebenda

ebenso

Bedingungen für die Versicherung zusätzlicher Gefahren zur Feuerversicherung für Industrie- und Handelsbetriebe

Bedingungen für die Versicherung zusätzlicher Gefahren zur FeuerBetriebsunterbrechungs-Versicherung für Industrie- und Handelsbetriebe

Einbruchdiebstahl

editor(s)

Einführungsgesetz bzw. Europäische Gemeinschaft(en) bzw. Erinnerungsgabe

Einführungsgesetz zum Bürgerlichen Gesetzbuch

Einführungsgesetz zum Gerichtsverfassungsgesetz v. 27.1.1877

Vertrag zur Gründung der Europäischen Gemeinschaft

Einführungsgesetz zum VVG

ehemalig

Einführung

eingehend

einschließlich

einschränkend

Einleitung

entgegen

Entscheidung

entsprechend

Entwurf

Erfurter Kommentar zum Arbeitsrecht, hrsg. von Dieterich/Hanau/

Schaub, 18. Aufl. (2018)

Ergebnis bzw. Ergänzung

Ergänzungsband

Erläuterung

Handkommentar zum Bürgerlichen Gesetzbuch, hrsg. von Westermann/Grunewald/Maier-Reimer, 15. Aufl. (2017)

Erwiderung

Einkommensteuergesetz

et cetera

Europäische Union

Gerichtshof der Europäischen Gemeinschaft

Entscheidungen des Gerichtshofs der Europäischen Gemeinschaften Amtliche Sammlung

Verordnung des Rates über die gerichtliche Zuständigkeit und die Anerkennung und Vollstreckung von Entscheidungen in Zivil- und Handelssachen (EG-Verordnung Nr. 44/2001)

Europarecht

europäisch

Vertrag über die Europäische Union (Lissabon-Vertrag)

Europäische Zeitschrift für Wirtschaftsrecht 
evtl.

EWG

EWGV

EWiR

EWR

f., ff.

FAG

Fahr/Kaulbach/Bähr/

Pohlmann/Bearbeiter

FamRZ

FAO

Farny

FBUB

Fenyves/Schauer/Bearbeiter

$\mathrm{FeV}$

FF

FG

FGG

FGO

FHB

FinDAG

Feyock/Jacobsen/Lemor/

Bearbeiter

Fn.

fragl.

FS

FZV

G

GB BAV

GB GDV

GBl.

GbR

GDV

GE

Geigel/Bearbeiter

gem.

GenG

GeschO

gesetzl.

GewArch

\section{GewO}

gg.

GG

ggf.

GKG

GKV

gl.

GmbHG

GmbHR eventuell

Europäische Wirtschaftsgemeinschaft

Vertrag zur Gründung der Europäischen Wirtschaftsgemeinschaft

Entscheidungen zum Wirtschaftsrecht

Europäischer Wirtschaftsraum

folgende

Gesetz über Fernmeldeanlagen

Versicherungsaufsichtsgesetz, 5. Aufl. (2012)

Ehe und Familie im privaten und öffentlichen Recht. Zeitschrift für das gesamte Familienrecht

Fachanwaltsordnung

Versicherungsbetriebslehre, 5. Aufl. (2011)

Allgemeine Feuer-Betriebsunterbrechungs-Versicherungsbedingungen

VersVG - Versicherungsvertragsgesetz, hrsg. von Fenyves/Schauer,

Loseblattwerk mit 3. Aktualisierung 2016

Fahrerlaubnis-Verordnung

Forum Familienrecht

Finanzgericht

Gesetz über die Angelegenheiten der freiwilligen Gerichtsbarkeit

Finanzgerichtsordnung

Feuerhaftungs-Versicherungsbedingung

Finanzdienstleistungsaufsichtsgesetz

Kraftfahrtversicherung, 3. Aufl. (2009)

Fußnote

fraglich

Festschrift

Fahrzeug-Zulassungsverordnung

Gesetz

Geschäftsbericht des Bundesaufsichtsamtes für das Versicherungswesen Geschäftsbericht des Gesamtverbandes der Deutschen Versicherungswirtschaft e.V.

Gesetzblatt

Gesellschaft bürgerlichen Rechts

Gesamtverband der Deutschen Versicherungswirtschaft e.V.

Geschäftsplanmäßige Erklärung

Haftpflichtprozess, hrsg. von Geigel, 27. Aufl. (2015)

gemäß

Gesetz betreffend die Erwerbs- und Wirtschaftsgenossenschaften

Geschäftsordnung

gesetzlich

Gewerbearchiv, Zeitschrift für Gewerbe- u. Wirtschaftsverwaltungsrecht

Gewerbeordnung

gegen

Grundgesetz für die Bundesrepublik Deutschland

gegebenenfalls

Gerichtskostengesetz

Gesetzliche Krankenversicherung

gleich

Gesetz betr. die Gesellschaften mit beschränkter Haftung

$\mathrm{GmbH}-\mathrm{Rundschau}$ (vorher: Rundschau für $\mathrm{GmbH}$ ) 
Verzeichnis der Abkürzungen und der abgekürzt zitierten Literatur

grdl.

grds.

Grimm

GrS

GrSZ

GRUR

GS

GüKG

GVBl.

GVG

GWB

grundlegend

grundsätzlich

Unfallversicherung: AUB, 5. Aufl. (2013)

Großer Senat

Großer Senat in Zivilsachen

Gewerblicher Rechtsschutz und Urheberrecht

Gedächtnisschrift

Güterkraftverkehrsgesetz

Gesetz- und Verordnungsblatt

Gerichtsverfassungsgesetz

Gesetz gegen Wettbewerbsbeschränkungen

Halbs.

Halm/Engelbrecht/Krahe

Halbsatz

Halm/Kreuter/Schwab/

Handbuch des Fachanwalts Versicherungsrecht, 6. Aufl. (2018)

Allgemeine Kraftfahrtbedingungen (AKB), hrsg. von Halm/Kreuter/ Schwab, 2. Aufl. (2015)

Bearbeiter

Hansen Beweislast

HansRGZ

HansRZ

Harbauer/Bearbeiter

HbgGarVO

Hdb.

$\mathrm{HdV}$

Hentschel/König/Dauer/

Bearbeiter

HGB

hins.

Hinw.

HK BGB/Bearbeiter

Beweislast und Beweiswürdigung im Versicherungsrecht (1990)

Hanseatische Rechts- und Gerichtszeitschrift

Hanseatische Rechtszeitschrift

Rechtsschutzversicherung. Kommentar zu den Allgemeinen Bedingungen für die Rechtsschutzversicherung (ARB), 8. Aufl. (2010)

Hamburger Verordnung über den Bau und Betrieb von Garagen und offenen Stellplätzen

Handbuch

Handwörterbuch der Versicherung, hrsg. von Farny/Helten/Koch/ Schmidt (1988)

Straßenverkehrsrecht, hrsg. von Hentschel/König/Dauer, 44. Aufl. (2017)

Handelsgesetzbuch

hinsichtlich

Hinweis

Bürgerliches Gesetzbuch Handkommentar, hrsg. von Schulze/Dörner/ Ebert et. al., 9. Aufl. (2016)

HK ZPO/Bearbeiter

h.A.

h.L.

h.M.

Hofmann

Hrsg./hrsg.

h.Rspr.

i.Allg.

IBR

i.d.F.

i.d.R.

i.d.S.

i.E.

i.e.S.

IFG

i.gl.S.

i.Grds.

IHK

Zivilprozessordnung Handkommentar, hrsg. von Saenger, 7. Aufl. (2017)

herrschende Ansicht, herrschende Auffassung

herrschende Lehre

herrschende Meinung

Schutzbriefversicherung (1996)

Herausgeber/herausgegeben

herrschende Rechtsprechung

im Allgemeinen

Immobilien \& Baurecht

in der Fassung

in der Regel

in diesem Sinne

im Ergebnis

im engeren Sinne

Informationsfreiheitsgesetz

im gleichen Sinne

im Grundsatz

Industrie- und Handelskammer

in Höhe von 
Verzeichnis der Abkürzungen und der abgekürzt zitierten Literatur

InfoV
inl.
insbes.
insges.
InsO
inzw.
i.R.d.
i.R.v.
i.S.
i.S.d.
i.S.e.
i.S.v.
i.techn.S.
i.U.
i.üb.
i.V.m.
i.w.
i.w.S.
i.Z.m.
JA
jew.
Jura
JurBüro
jurisPK/Bearbeiter
jurisPR
JuS
JW
JZ

KalV

Kap.

Kaulbach/Bähr/Pohlmann

$\mathrm{Kfz}$

KfzEFondsV

\section{KfzPflVV}

$\mathrm{KfzSBHH}$

KG

$\mathrm{KH}$

KK-OWiG/Bearbeiter

Kl.

KomE

$\mathrm{K} \& \mathrm{R}$

krit.

KritVj

KStG siehe VVG-Info V

inländisch

insbesondere

insgesamt

Insolvenzordnung

inzwischen

im Rahmen der/des

im Rahmen von

im Sinne

im Sinne der/des

im Sinne einer(s)

im Sinne von

im technischen Sinne

im Unterschied

im Übrigen

in Verbindung mit

im Wesentlichen

im weiteren Sinne

im Zusammenhang mit

Juristische Arbeitsblätter für Ausbildung und Examen

jeweils

Juristische Ausbildung

Das Juristische Büro

juris Praxiskommentar BGB, hrsg. von Herberger/Martinek/Rüßmann/Weth, 8. Aufl. (2017)

juris PraxisReport

Juristische Schulung. Zeitschrift für Studium und Ausbildung

Juristische Wochenschrift

Juristenzeitung

Verordnung über die versicherungsmathematischen Methoden zur Prämienkalkulation und zur Berechnung der Alterungsrückstellung in der privaten Krankenversicherung (Kalkulationsverordnung - KalV) Kapitel

Versicherungsaufsichtsgesetz, 6. Aufl. (2018)

Kraftfahrzeug

Verordnung über den Entschädigungsfonds für Schäden aus Kraftfahrzeugunfällen

Kraftfahrzeugpflichtversicherungsverordnung

Sonderbedingungen zur Kfz-Haftpflicht- und Kaskoversicherung für Kfz-Handel und -Handwerk

Kammergericht, Kommanditgesellschaft

Kraftfahrzeug-Haftpflicht

Karlsruher Kommentar zum Gesetz über Ordnungswidrigkeiten, 5. Aufl. (2018)

Klausel bzw. Kläger/in

Kommissionsentwurf zur Reform des Versicherungsvertragsrechts; zitiert nach: Abschlussbericht der Kommission zur Reform des Versicherungsvertragsrechts vom 19. April (2004), hrsg. von Egon Lorenz (2004)

Kommunikation und Recht

kritisch

Kritische Vierteljahresschrift für Gesetzgebung und Rechtsprechung Körperschaftsteuergesetz 
Verzeichnis der Abkürzungen und der abgekürzt zitierten Literatur

Lackner/Kühl
Langheid/Rixecker
(vormals Römer/Langheid)
Langheid/Wandt/Bearbeiter

LG
l.Sp.
lit.
Lit.
LM
LMK
Looschelders/Pohlmann/
Bearbeiter
Looschelders/Pfaffenholz/
Bearbeiter
LS
lt.
LugÜ

m.

\section{Martin SVR}

MAH Versicherungsrecht/

Bearbeiter

Maunz/Dürig/Bearbeiter

m.a.W.

m.Bespr.

MBKK

MBKT

MBPPV

MBUB

$\mathrm{MdB}$

MDR

missverst.

m.krit.Anm.

MMR

MMW

MontÜG

Motive

MÜ

MünchKomm-AktG/

Bearbeiter

MünchKomm-BGB/

Bearbeiter

MünchKomm-UWG/

Bearbeiter
StGB, 29. Aufl. (2018)

Versicherungsvertragsgesetz, 5. Aufl. (2016)

Münchener Kommentar Versicherungsvertragsgesetz: VVG; Band 1: \$S 1-99 VVG und VVG-InfoV, 2. Auflage (2016); Band 2: $\mathbb{\$} \mathbb{1 0 0 - 2 1 6}$ VVG, 2. Auflage (2017); Band 3: Nebengesetze, Systematische Darstellungen, 2. Auflage (2017)

Landgericht

linke Spalte

littera (Buchstabe)

Literatur

Nachschlagewerk des Bundesgerichtshofs, hrsg. von Lindenmaier/ Möhring u.a. (zit. nach Paragraph u. Nummer)

Kommentierte BGH-Rechtsprechung Lindenmaier-Möhring

VVG Versicherungsvertragsgesetz, Kommentar, 3. Aufl. (2017)

ARB - Allg. Bedingungen für die Rechtsschutzversicherung, Kommen$\operatorname{tar}(2014)$

Leitsatz

laut

Übereinkommen über die gerichtliche Zuständigkeit und die Anerkennung und Vollstreckung von Entscheidungen in Zivil- und Handelssachen (Lugano-Übereinkommen)

mit

Sachversicherungsrecht, Kommentar, 3. Aufl. (1992)

Münchener Anwaltshandbuch Versicherungsrecht, hrsg. von Höra, 4. Auflage (2017)

Grundgesetz, Loseblatt-Kommentar, 82. Ergänzungslieferung (1/2018), begr. von Maunz/Dürig

mit anderen Worten

mit Besprechung

Musterbedingungen für die Krankheitskosten- und Krankenhaustagegeldversicherung

Musterbedingungen für die Krankentagegeldversicherung

Musterbedingungen für die private Pflegeversicherung

Allgemeine Maschinen-Betriebsunterbrechungs-Versicherungsbedingungen

Mitglied des Bundestags

Monatsschrift für Deutsches Recht

missverständlich

mit kritischer Anmerkung (von)

MultiMedia und Recht

Münchner Medizinische Wochenschrift

Montrealer-Übereinkommen-Durchführungsgesetz vom 6.4.2004

Motive zum VVG, Nachdruck (1963)

Montrealer Übereinkommen (Übereinkommen zur Vereinheitlichung bestimmter Vorschriften über die Beförderung im internationalen Luftverkehr vom 28.5.1999)

Münchener Kommentar zum Aktiengesetz, hrsg. von Goette/Habersack/Kalss, 4. Aufl. (2014ff.)

Münchener Kommentar zum Bürgerlichen Gesetzbuch, hrsg. von

Rebmann/Säcker/Rixecker, 7. Aufl. (2015ff.)

Münchener Kommentar zum Lauterkeitsrecht, hrsg. von Heermann/ Schlingloff, 2. Aufl. (2014) 
MünchKomm-ZPO/ Bearbeiter

MünchKomm-StGB/ Bearbeiter

MünchKomm-StVR

Musielak/Bearbeiter

m.w.N.

m.W.v.

m.zust.Anm.

$\mathrm{N}$.

Nachtr.

n.F.

NJ

NJOZ

NJW

NJWE-VHR

NJW-RR

NK-BGB/Bearbeiter

Nr.

NStZ

NTS

NTS-ZA

NVersZ

NVwZ

NZA

NZG

NZI

NZS

NZV

o.

o.ä.

ob.dict.

Oetker/Bearbeiter

ÖBGBl

öffentl.

o.g.

ÖOGH

OHG

OLG

OLGZ

OVG

OWiG

Palandt/Bearbeiter

PartGG

PflVG

PfP-Truppenstatut

PHi
Münchener Kommentar zur Zivilprozessordnung mit Gerichtsverfassungsgesetz und Nebengesetzen, hrsg. von Krüger/Rauscher, 5. Aufl. (2016f.)

Münchener Kommentar zum Strafgesetzbuch, hrsg. von Joecks/Miebach, 3. Aufl. (2016ff.)

Münchener Kommentar zum Straßenverkehrsrecht, hrsg. von König, Bd. 1 (2016)

Kommentar zur Zivilprozessordnung, 15. Aufl. (2018)

mit weiteren Nachweisen

mit Wirkung vom

mit zustimmender Anmerkung

Nachweise

Nachtrag

neue Fassung

Neue Justiz

Neue Juristische Online Zeitung

Neue Juristische Wochenschrift

NJW-Entscheidungsdienst Versicherungs-/Haftungsrecht

NJW-Rechtsprechungs-Report Zivilrecht

NomosKommentar BGB, hrsg. von Dauner-Lieb/Heidel/Ring, 6 Bände (2014ff.) (ehemaliger AnwaltKommentar BGB)

Nummer

Neue Zeitschrift für Strafrecht

Nato-Truppen-Statut

Nato-Truppenstatut-Zusatzabkommen

Neue Zeitschrift für Versicherung und Recht

Neue Zeitschrift für Verwaltungsrecht

Neue Zeitschrift für Arbeits- und Sozialrecht

Neue Zeitschrift für Gesellschaftsrecht

Neue Zeitschrift für Insolvenzrecht

Neue Zeitschrift für Sozialrecht

Neue Zeitschrift für Verkehrsrecht

oben

oder ähnlich

obiter dictum

Handelsgesetzbuch, 5. Aufl. (2017)

österreichisches Bundesgesetzblatt

öffentlich

oben genannt

Österreichischer Oberster Gerichtshof

Offene Handelsgesellschaft

Oberlandesgericht

Entscheidungen der Oberlandesgerichte in Zivilsachen, einschließlich der freiwilligen Gerichtsbarkeit

Oberverwaltungsgericht

Gesetz über Ordnungswidrigkeiten

Bürgerliches Gesetzbuch, 77. Aufl. (2018)

Partnerschaftsgesellschaftsgesetz

Pflichtversicherungsgesetz

Übereinkommen zwischen den Vertragsstaaten des Nordatlantikvertrags und den anderen an der Partnerschaft für den Frieden teilnehmenden Staaten über die Rechtsstellung ihrer Truppen (PfP-Truppenstatut) Haftpflicht international (vormals Produkthaftpflicht international) 
PKV

polit.

ProdHM

Prölss/Martin/Bearbeiter
Prölss/Dreher/Bearbeiter
PStG
psych.

RAA

RBerG

RdA

RdErl.

RDG

RdK

RDV

RdW

rechtspol.

rechtsvergl.

RefE

ReformG

Reg.

RegE

RegBl.

rel.

RG

RGBl.

RGRK/Bearbeiter

RGZ

RHG

RiL

Rn.

Rom I-VO

Rom II-Verordnung

Römer/Langheid
Rpfleger
RpflG
Rspr.
RStBl.
RT
RTDrucks.

Private Krankenversicherung

politisch

Besondere Bedingungen und Risikobeschreibungen für die Produkthaftpflichtversicherung von Industrie- und Handelsbetrieben

Versicherungsvertragsgesetz, 30. Aufl. (2018)

Versicherungsaufsichtsgesetz, hrsg. von Dreher, 13. Aufl. (2018)

Personenstandsgesetz

psychisch

Reichsaufsichtsamt für Privatversicherung

Rechtsberatungsgesetz (bis 1962: Gesetz zur Verhütung von Mißbräu-

chen auf dem Gebiete der Rechtsberatung)

Recht der Arbeit

Runderlaß/Runderlass

Rechtsdienstleistungsgesetz

Das Recht des Kraftfahrers, Unabhängige Monatsschrift des Kraftverkehrsrechts (1926-43, 1949-55)

Recht der Datenverarbeitung

Recht der Wirtschaft (Österreich)

rechtspolitisch

rechtsvergleichend

Referentenentwurf des Bundesministeriums der Justiz zur Reform des Versicherungsvertragsrechts mit Begründung (nicht veröffentlicht; zitiert nach der vom BMJ online zur Verfügung gestellten PDF-Datei; u.a. noch abrufbar unter: http://www.brak.de/seiten/pdf/aktuelles/ versicherungsvertragsrecht.pdf)

Gesetz zur Reform des Versicherungsvertragsrechts vom 23.11.2007 (BGBl. I S. 2631) (siehe auch VVG-Reform 2008)

Regierung

Regierungsentwurf eines Gesetzes zur Reform des Versicherungsvertragsrechts (BTDrucks. 16/3945); siehe auch Ausschussbericht

Regierungsblatt

relativ

Reichsgericht

Reichsgesetzblatt

Reichsgerichtsrätekommentar - Das Bürgerliche Gesetzbuch. Kommentar, hrsg. von den Mitgliedern des Bundesgerichtshofs, 12. Aufl. (1975 ff.)

Entscheidungen des Reichsgerichts in Zivilsachen (zit. nach Band u. Seite)

Reichshaftpflichtgesetz

Richtlinie

Randnummer(n)

Rom I-Verordnung (Verordnung (EG) Nr. 593/2008 des Europäischen Parlaments und des Rates über das auf vertragliche Schuldverhältnisse anzuwendende Recht)

Rom II-Verordnung (Verordnung (EG) Nr. 864/2007 des Europäischen Parlaments und des Rates über das auf außervertragliche Schuldverhältnisse anzuwendende Recht)

siehe Langheid/Rixecker

Der Deutsche Rechtspfleger

Rechtspflegergesetz

Rechtsprechung

Reichssteuerblatt

Reichstag

Drucksachen des Reichstags 
r.Sp.

RuS

Rüffer/Halbach/

Schimikowski/Bearbeiter

RVerkBl.

RVG

RVO

s.

S.

s.a.

Sachs/Bearbeiter

$\mathrm{SB}$

ScheckG

SchiedsVZ

Schönke/Schröder

Schwintowski/Brömmel-

meyer/Bearbeiter

Sen.

Seuff. Arch.

SF

SGB I, IV, V, VIII, IX, X, XI

SGb.

SGG

SGIN

SkAufG

s.o.

Soergel/Bearbeiter

sog.

SP

spez.

$\mathrm{SpV}$

Stadler/Gail

Staudinger/Bearbeiter

Stein/Jonas/Bearbeiter

StGB

Stiefel/Maier

StPO

str.

st.Rspr.

StuR rechte Spalte

Recht und Schaden

Versicherungsvertragsgesetz Handkommentar, hrsg. von Rüffer/Halbach/Schimikowski, 3. Aufl. (2015)

Reichsverkehrsblatt

Rechtsanwaltsvergütungsgesetz

Reichsversicherungsordnung

siehe

Satz, Seite

siehe auch

Grundgesetz, Kommentar, hrsg. von Sachs, 8. Aufl. (2018)

Selbstbeteiligung

Scheckgesetz

Zeitschrift für Schiedsverfahren - German Arbitration Journal

Strafgesetzbuch, Kommentar, 29. Aufl. (2014)

Praxiskommentar zum Versicherungsvertragsrecht, 3. Aufl. (2017)

Senat

Seuffert 's Archiv für die Entscheidungen der obersten Gerichte in den deutschen Staaten, (8.1855)

Schadensfreiheit

I: Sozialgesetzbuch, Allg. Teil

IV: Sozialgesetzbuch, Gemeinsame Vorschriften für die Sozialversicherung

V: Sozialgesetzbuch, Gesetzliche Krankenversicherung

VIII: Sozialgesetzbuch, Kinder- und Jugendhilfe

IX: Sozialgesetzbuch, Rehabilitation und Teilhabe von Menschen mit Behinderungen

X: Sozialgesetzbuch, Verwaltungsverfahren, Zusammenarbeit der Leistungsträger und ihre Beziehung zu Dritten

XI: Soziale Pflegeversicherung

Sozialgerichtsbarkeit/Die Sozialgerichtsbarkeit (Zeitschrift)

Sozialgerichtsgesetz

Sonderbedingungen für die gleitende Neuwertversicherung von Wohn-, Geschäfts- und landwirtschaftlichen Gebäuden

Streitkräfteaufenthaltsgesetz

siehe oben

Bürgerliches Gesetzbuch, 13. Aufl. (2000ff.)

sogenannt(e)

Schaden-Praxis

speziell

Spektrum für Versicherungsrecht

Die Kfz-Versicherung (2015)

Kommentar zum Bürgerlichen Gesetzbuch mit Einführungsgesetz und Nebengesetzen, 13. Bearbeitung (1993ff.)

Kommentar zur Zivilprozessordnung, 23. Aufl. (2013ff.)

Strafgesetzbuch

Kraftfahrtversicherung. Kommentar zu den Allgemeinen Bedingungen für die Kraftfahrtversicherung - AKB sowie zu weiteren Gesetzes- und Regelwerken in der Kraftfahrtversicherung, 19. Aufl. (2017)

Strafprozessordnung

strittig, streitig

ständige Rechtsprechung

Staat und Recht 
Verzeichnis der Abkürzungen und der abgekürzt zitierten Literatur

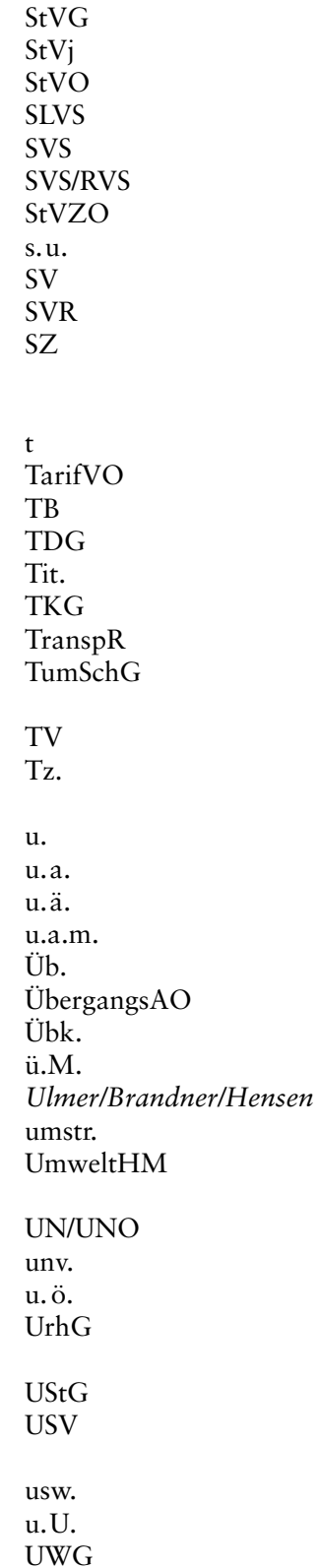

VA

VAG

v.A.w.

VD

VE
Straßenverkehrsgesetz

Steuerliche Vierteljahresschrift

Straßenverkehrsordnung

Speditions-, Logistik- und Lagerversicherungsschein

Speditions-Versicherungsschein

Speditions- und Rollfuhr-Versicherungsschein

Straßenverkehrs-Zulassungs-Ordnung

siehe unten

Sachverhalt

Straßenverkehrsrecht

Entscheidungen des Österreichischen Obersten Gerichtshofes in Zivilund Justizverwaltungssachen

Tonne

Verordnung über die Tarife in der Kfz-Haftpflichtversicherung

Tarifbestimmung

Gesetz über die Nutzung von Telediensten

Titel

Telekommunikationsgesetz

Transportrecht

Gesetz über die durch innere Unruhen verursachten Schäden vom 12.5.1920

Truppenvertrag

Textzahl

unten/und

unter anderem

und ähnlich

und anderes mehr

Überblick, Übersicht

Übergangsanordnung

Übereinkommen

überwiegende Meinung

AGB-Recht, 12. Aufl. (2016)

umstritten

Besondere Bedingungen und Risikobeschreibungen für die Versicherung der Haftpflicht wegen Schäden durch Umwelteinwirkung

United Nations Organization (Vereinte Nationen)

unveröffentlicht

und öfter

Gesetz über Urheberrecht und verwandte Schutzrechte (Urheberrechtsgesetz)

Umsatzsteuergesetz

Allgemeine Versicherungsbedingungen für die Umweltschadensversicherung

und so weiter

unter Umständen

Gesetz gegen den unlauteren Wettbewerb

Veröffentlichungen des Reichsaufsichtsamtes für Privatversicherung, ab 1947: ... des Zonenamtes des Reichsaufsichtsamtes für das Versicherungswesen (Hamburg)

Gesetz über die Beaufsichtigung der Versicherungsunternehmungen von Amts wegen

Verkehrsdient

Vorentwurf 
Veith/Gräfe/Bearbeiter VerBAV/VerBaFin

VerfGH
VerglO
Verh.
VerkMitt
vermitt.
VersG
VersAG
VersArch
VersM
VersPrax, VP
VersR

VersRAI

VersRdsch.

VersVermV

VersVG

VersWissArch

VersWiss. Stud.

VerwArch.

VG

VGB

VGB 2008, 2010

$\mathrm{VGH}$

vgl.

VGS

VHB

VHB 2008

VHV

VIZ

$\mathrm{VN}$

VO

VOBl.

VomVO

vorangeh.

Voraufl.

Vorbem.

vorgen.

VRR

VR

VRS

VU

VuR

VVaG
Versicherungsprozess, 3. Aufl. (2016)

Veröffentlichungen des Bundesaufsichtsamtes für das Versicherungsund Bausparwesen, ab 1973: ... des Bundesaufsichtsamtes für das Versicherungswesen,

ab Mai 2002: VerBAFin = Veröffentlichungen der Bundesanstalt für Finanzdienstleistungsaufsicht (Versicherungsbereich)

Verfassungsgerichtshof

Vergleichsordnung

Verhandlungen des Deutschen Bundestages (BT), des Deutschen Juristentages (DJT) usw.

Verkehrsrechtliche Mitteilungen

vermittelnd

Gesetz über Versammlungen und Aufzüge (Versammlungsgesetz)

Versicherungsaktiengesellschaft

Versicherungsarchiv

Versicherungsmedizin

Die Versicherungspraxis

Versicherungsrecht. Zeitschrift für Versicherungsrecht, Haftungs- und Schadensrecht

Versicherungsrecht. Beilage Ausland

Versicherungsrundschau (Österreich)

Verordnung über die Versicherungsvermittlung und -beratung

österreichisches Versicherungsvertragsgesetz

Versicherungswissenschaftliches Archiv

Versicherungswissenschaftliche Studien, hrsg. von Brömmelmeyer et. al.

Verwaltungsarchiv

Verwaltungsgericht

Allgemeine Bedingungen für die Neuwertversicherung von Wohngebäuden gegen Feuer-, Leitungswasser- und Sturmschäden

Allgemeine Wohngebäude-Versicherungsbedingungen

Verwaltungsgerichtshof

vergleiche

Vereinigter Großer Senat

Allgemeine Bedingungen für die Neuwertversicherung des Hausrats gegen Feuer-, Einbruchdiebstahl-, Beraubungs-, Leitungswasser-, Sturm- und Glasbruchschäden/Allgemeine Hausratversicherungsbedingungen

Allgemeine Hausrat-Versicherungsbedingungen

Verkehrshaftungsversicherung

Zeitschrift für Vermögens- und Immobilienrecht

Versicherungsnehmer/in

Verordnung

Verordnungsblatt

Verfahrensordnung des Versicherungsombudsmanns

vorangehend

Vorauflage

Vorbemerkung

vorgenannt

Verkehrsrechtliche Rundschau

Versicherer

Verkehrsrechts-Sammlung, Entscheidungen aus allen Gebieten des

Verkehrsrechts (zit. nach Band u. Seite)

Versicherungsunternehmen

Verbraucher und Recht

Versicherungsverein auf Gegenseitigkeit 
VVG

VVG-InfoVO

VVGE

VVG-Kommission
VVGRefG $b z w$.
VVG-Reform 2008
VVV

VW

VwGO

$\mathrm{VwV}$

$\mathrm{VwVfG}$

VwVG

VwZG

WaffG

Wandt

weitergeh.

WM

Wolf/Lindacher/Pfeiffer

WRP

WuM

Wussow

(Z)

z.B.

ZEuP

ZfRV

ZfS/zfs

$\mathrm{ZfV}$

ZGR

Ziff.

ZIP

zit.

ZKM

ZMR

Zöller/Bearbeiter

ZollG

$\mathrm{ZPO}$

ZRP

ZSW

z.T.

ZusBedIT

zusf.

zust.

ZustG

zutr.

z.V.b.

ZVersWiss
Gesetz über den Versicherungsvertrag (Versicherungsvertragsgesetz) Verordnung über Informationspflichten bei Versicherungsverträgen Entscheidungssammlung zum Versicherungsvertragsrecht (VVGE): Entscheidungen zum Versicherungsvertragsgesetz (VVG) und zu den Allgemeinen Versicherungsbedingungen (AVB), hrsg. von Dietrich Müller

Kommission zur Reform des Versicherungsvertragsrechts

Gesetz zur Reform des Versicherungsvertragsrechts vom 23.11.2007 (BGBl. I S. 2631) (siehe auch ReformG)

Versicherungswissenschaft, Versicherungspraxis, insbesondere Versicherungsmedizin (später DVZ)

Versicherungswirtschaft

Verwaltungsgerichtsordnung

Verwaltungsvorschrift

Verwaltungsverfahrensgesetz

Verwaltungsvollstreckungsgesetz

Verwaltungszustellungsgesetz

Waffengesetz

Versicherungsrecht, 6. Aufl. (2016)

weitergehend

Wertpapier-Mitteilungen

AGB-Recht, Kommentar, 6. Aufl. (2013)

Wettbewerb in Recht und Praxis

Wohnungswirtschaft und Mietrecht

Unfallhaftpflichtrecht, 16. Aufl. (2014)

Entscheidung in Zivilsachen

zum Beispiel

Zeitschrift für Europäisches Privatrecht

Zeitschrift für Rechtsvergleichung, Internationales Privatrecht u. Europarecht

Zeitschrift für Schadensrecht

Zeitschrift für Versicherungswesen

Zeitschrift für Unternehmens- und Gesellschaftsrecht

Ziffer

Zeitschrift für Wirtschaftsrecht

zitiert

Zeitschrift für Konfliktmanagement

Zeitschrift für Miet- und Raumrecht

Zivilprozessordnung mit Gerichtsverfassungsgesetz und Nebengesetzen; Kommentar 32. Aufl. (2018)

Zollgesetz

Zivilprozessordnung

Zeitschrift für Rechtspolitik

Zeitschrift für das gesamte Sachverständigenwesen

zum Teil

Zusatzbedingungen zur Betriebshaftpflichtversicherung für die Nutzer von Internet-Technologien

zusammenfassend

zustimmend

Zustimmungsgesetz

zutreffend

zur Veröffentlichung bestimmt

Zeitschrift für die gesamte Versicherungswissenschaft (zitiert nach Jahr und Seite) 
Verzeichnis der Abkürzungen und der abgekürzt zitierten Literatur

ZVG

ZVR

Zw.

$\mathrm{zz}$.

ZZP

ZZPInt
Gesetz über die Zwangsversteigerung und die Zwangsverwaltung (Zwangsversteigerungsgesetz)

Zeitschrift für Verkehrsrecht

zweifelhaft

zurzeit

Zeitschrift für Zivilprozess

Zeitschrift für Zivilprozess International 
